This item was submitted to Loughborough's Research Repository by the author.

Items in Figshare are protected by copyright, with all rights reserved, unless otherwise indicated.

\title{
Characterization of cadmium sulfide buffer layers using electrolyte contacts
}

PLEASE CITE THE PUBLISHED VERSION

https://doi.org/10.1016/j.tsf.2018.12.024

PUBLISHER

(c) Elsevier

VERSION

AM (Accepted Manuscript)

PUBLISHER STATEMENT

This paper was accepted for publication in the journal Thin Solid Films and the definitive published version is available at https://doi.org/10.1016/j.tsf.2018.12.024

\section{LICENCE}

CC BY-NC-ND 4.0

\section{REPOSITORY RECORD}

Deng, Wentao, Laurence M. Peter, Fabiana Lisco, and Jake Bowers. 2019. "Characterization of Cadmium Sulfide Buffer Layers Using Electrolyte Contacts”. figshare. https://hdl.handle.net/2134/36679. 


\title{
Characterization of Cadmium Sulfide Buffer Layers using Electrolyte Contacts
}

Wentao Deng ${ }^{1}$, Laurence Peter ${ }^{1, *},{\text { Fabiana } \text { Lisco }^{2} \text { and Jake Bowers }}^{2}$

1. Department of Chemistry, University of Bath, Bath BA2 7AY, UK

2. Centre for Renewable Energy Systems Technology (CREST), School of Electronic, Electrical and Systems Engineering, Loughborough University, Leicestershire, LE11 3TU, UK

* Corresponding author: 1.m.peter@bath.ac.uk

\begin{abstract}
Electrolyte contacts were used to characterize cadmium sulfide (CdS) films grown by chemical bath deposition on fluorine-doped tin oxide glass (FTO). Capacitance measurements were made in a buffered electrolyte free of redox species, and cyclic sweep voltammetry was carried out using an electrolyte containing $\mathrm{Fe}(\mathrm{CN})_{6}{ }^{4-}$ ions. A theoretical model was developed to describe the influence of pinholes on Mott Schottky plots of CdS-coated FTO. The model allows estimation of the pinhole coverage by fitting the Mott Schottky plot over an extended voltage range. Cyclic voltammetry in a hexacyanoferrate(II) electrolyte also allows detection of pinholes, but quantitative estimation of pinhole coverage is complicated by the fact that diffusion of $\mathrm{Fe}(\mathrm{CN})_{6}{ }^{4-}$ to pinholes occurs by hemispherical diffusion, which can substantially enhance the current response. Comparison of the results of capacitance and voltammetry measurements provides insights into average pore size and number density. Results presented for CdS films grown using two different chemical bath compositions reveal substantial differences in pinhole numbers and doping density.
\end{abstract}




\section{Introduction}

Cadmium sulfide $(\mathrm{CdS})$ buffer layers are used thin film solar cells in both superstrate (e.g. CdTe) and substrate (e.g. CIGS) cell configurations. The most common deposition method involves using a chemical bath containing thiourea as a sulfur source, a cadmium salt and ammonia with or without ammonia salts (chemical bath deposition: CBD). Chloride ions may also be added to increase the donor concertation in the film. The substrate in the superstrate cell configuration is a transparent conducting oxide (TCO) such as fluorine-doped tin oxide (FTO) on glass. In the case of substrate cells, the CdS layer is deposited on the absorber layer. The quality of the CdS layers in both configurations is critical for cell efficiency. Not only the electronic properties (doping density, band edge mismatch) but also the structural integrity of the films is important since pinholes offer pathways for shunting the cell, leading to losses. Consequently, it is important to develop substrate cleaning [1] and CBD protocols that minimize pinholes as far as possible and control the doping density. Larger pinholes in $\mathrm{CdS}$ films on FTO can be detected by optical microscopy using blue light [2], but the approach outlined in the present paper is a relatively simple alternative.

The kinetics and mechanism of CdS deposition have been studied extensively over the last 25 years. The early fundamental work of the Lincot group [3-5] is particularly noteworthy since dynamic in situ measurements during growth using a quartz crystal microbalance (QCM) and impedance revealed details of the different stages of the deposition process. Differences between the film thickness evaluated from QCM measurements and from capacitance were attributed to a dual structure of the CdS film, with an inner compact layer and an outer more porous layer that develops at longer deposition times.

Inert electrolytes can be used to form non-destructive conformal contacts to thin semiconductor films. Our group, in collaboration with BP Solar, used impedance measurements with electrolyte contacts to characterize CdS films deposited on FTO for the 
fabrication of CdTe solar cells [6]. This work showed that doping density, film thickness and surface roughness can be extracted from the voltage dependence of the capacitance measured under reverse bias in the dark. The electrolyte acts as an electronic blocking contact, and the band bending in the Cds buffer layer and underlying FTO can be controlled by varying the electrode potential relative to the flatband potential. Mott Schottky plots of $\mathrm{C}^{-2}$ vs. electrode potential show a transition from a high slope to an almost flat region at a transition voltage that corresponds to the point at which the space charge region extends to the back of the CdS film and begins to enter the much more highly doped FTO layer as illustrated in Figure 1. At this transition voltage, the $\mathrm{CdS}$ film is fully depleted, and the measured capacitance corresponds to the geometric capacitance $C_{g e o}=\varepsilon_{0} \varepsilon_{C d S} / d_{C d S}$, which depends only on the film thickness $d_{C d S}$ and the relative permittivity $\varepsilon_{C d S}$. At higher voltages, the slope of the Mott Schottky plot is expected to be determined by the doping density of the FTO, which is typically at least 4 orders of magnitude higher than that of the CBD CdS layer. For this reason, the Mott Schottky plot should show a transition to an essentially horizontal plateau (this situation has been considered by van de $\mathrm{Krol}$ et al. for the case of $\mathrm{TiO}_{2}$ films on FTO [7]). However, the slope of experimental Mott Schottky plots for CdS-coated FTO beyond the transition is often higher than expected, and the present study provides evidence that this is because the CdS films contain pores that expose the underlying FTO to the electrolyte. We show that an analysis of the capacitance behaviour in this case allows determination of the exposed area associated with pinholes in the CdS.

The porosity of $\mathrm{CdS}$ films has also been estimated using electrolytes that contain suitable redox species such as $\mathrm{Fe}(\mathrm{CN})_{6}^{4-}$ [8]. This is possible because $\mathrm{CdS}$ is an n-type semiconductor, so that oxidation of $\mathrm{Fe}(\mathrm{CN})_{6}{ }^{4-}$ is not possible in the dark because the hole concentration is negligible. However, electrochemical oxidation of $\mathrm{Fe}(\mathrm{CN})_{6}{ }^{4-}$ to $\mathrm{Fe}(\mathrm{CN})_{6}{ }^{3-}$ is facile at FTO, so that any pinholes will give rise to an oxidation current when the electrode 
potential is scanned into the appropriate region. Since pinholes will behave as an array of FTO ultramicroelectrodes, it is necessary to consider the hemispherical diffusion geometry that enhances the flux of $\mathrm{Fe}(\mathrm{CN})_{6}{ }^{4-}$ to the pinholes. The present study compared the determination of pinhole area by the two methods: capacitance-voltage measurements with an inert electrolyte and cyclic voltammetry with an electrolyte containing $\mathrm{Fe}(\mathrm{CN})_{6}{ }^{4-}$ ions. The results reveal significant differences in pinhole coverage for different CBD protocols.

\section{Theory}

\section{Capacitance voltage measurements}

The capacitance voltage behaviour of the CdS/electrolyte junction is given by the Mott Schottky equation, which takes the form

$$
\frac{1}{c^{2}}=\frac{1}{C_{H}^{2}}+\frac{1}{C_{s c, C d S}^{2}}=\frac{1}{C_{H}^{2}}+\left(\frac{2}{\varepsilon_{C d S} \varepsilon_{0} q N_{d, C d S}}\right)\left(E-E_{f b, C d S}-\frac{k_{B} T}{q}\right)
$$

Here $C_{H}$ is the capacitance of the Helmholtz double layer, $C_{s c, C d S}$ is the space charge capacitance of the CdS layer, $\varepsilon_{C d S}$ is the relative permittivity of CdS (taken as 8.9 ), $N_{d, C d S}$ is the donor density in the CdS (assumed to be uniform) and $E_{f b, C d S}$ is the flat band potential of the CdS in the electrolyte used (in our case phosphate buffer, $\mathrm{pH} 7$ ).

In principle, the doping density can be determined from the slope, $S$, of the Mott Schottky plot of $1 / C^{2}$ vs. potential.

$$
S=\left(\frac{2}{\varepsilon_{C d S^{2} \varepsilon_{0} q N_{d, C d S}}}\right)
$$

However, equation 1 only applies if $W_{s c}$, the width of the space charge region in the CdS, is less than the CdS film thickness $d_{C d S}$.

$$
W_{s c}=\left(\frac{2 \varepsilon_{C d S} \varepsilon_{0}}{q N_{d . C d S}}\right)^{1 / 2}\left(E-E_{f b, C d S}-\frac{k_{B} T}{q}\right)^{1 / 2}
$$


For higher applied voltages, the space charge region extends into the underlying FTO as shown in Figure 1.
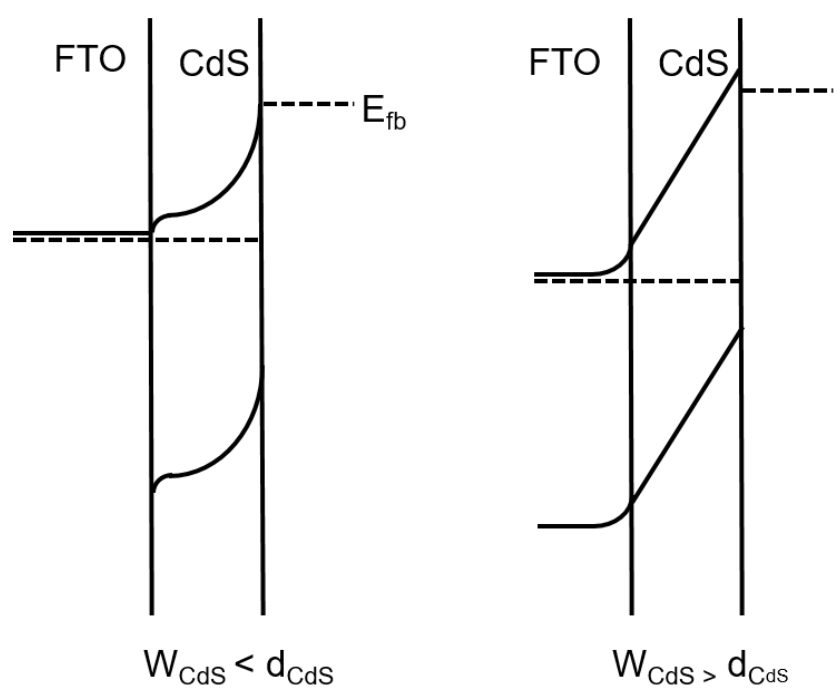

Figure 1. Band bending diagram showing the transition from the situation where the space charge is located only in the CdS to the situation where the space charge extends into the underlying FTO. For simplicity, only the conduction band of the wide bandgap oxide is shown. $\mathrm{W}_{\mathrm{CdS}}$ is given by equation 3 .

We can therefore define the transition voltage $V_{\text {trans }}$ at which this occurs by the condition

$$
W_{s c}=d_{C d S} \text { for }\left(E-E_{f b, C d S}-\frac{k_{B} T}{q}\right)=V_{\text {trans }}
$$

In practice, the transition is not abrupt since the film and substrate are non-uniform, but $V_{\text {trans }}$ can be estimated from the intersection point obtained by linear extrapolation of the two linear regions in the Mott Schottky plot. $V_{\text {trans }}$ can then be used to estimate the thickness of the CdS film since

$$
d_{C d S}=\left(V_{\text {trans }}-\frac{k_{B} T}{q}\right)^{1 / 2} \varepsilon_{C d S} \varepsilon_{0} S^{1 / 2}
$$

For voltages higher than $V_{\text {trans, }}$, the space charge region enters the underlying FTO, and as equation 6 shows, the slope of the Mott Schottky plot is determined only by the donor density of the FTO [7].

$$
\frac{1}{C^{2}}=\left(1-\frac{\varepsilon_{F T O} N_{d, C d S}}{\varepsilon_{C d S} N_{d . F T O}}\right)\left(\frac{d_{C d S}^{2}}{\varepsilon_{C d S}}\right)+\left(\frac{2}{\varepsilon_{F T O} \varepsilon_{0} q N_{d, F T O}}\right)\left(E-E_{f b, C d S}\right)
$$


We now consider the effect of pinholes on the Mott Schottky plot. We assume that the pinholes penetrate through the CdS film, exposing the bare FTO substrate. The exposed FTO gives rise to a capacitance that appears in parallel with that of the CdS film. The fraction of exposed area is taken as $\theta$. The parallel capacitance due to pinholes can be obtained from the appropriate Mott Schottky expression for the FTO substrate in contact with the electrolyte scaled by $\theta$, the relative area of the FTO exposed by pinholes.

$$
\frac{1}{c^{2}}=\frac{1}{C_{H}^{2}}+\frac{1}{C_{S c, F T O}^{2}}=\frac{1}{C_{H}^{2}}+\left(\frac{2}{\varepsilon_{F T O} \varepsilon_{0} q N_{d, F T O}}\right)\left(E-E_{f b, F T O}-\frac{k_{B} T}{q}\right)
$$

The values of $N_{d, F T O}$ and $E_{f b, F T O}$ in equation 7 are obtained from Mott Schottky plots measured for bare FTO in the same electrolyte.

Since the FTO capacitance due to pinholes appears in parallel with the capacitance of the CdS film, the capacitance per unit area of the FTO is scaled by $\theta$ and added to the capacitance given by either equation 1 or equation 6 in the voltage ranges below and above $V_{\text {trans }}$ respectively to give the total capacitance of a pinholed film.

The calculated effect of pinholes on the Mott Schottky plot for a $50 \mathrm{~nm}$ CdS film is illustrated in Figure 2 for different values of $\theta$. 


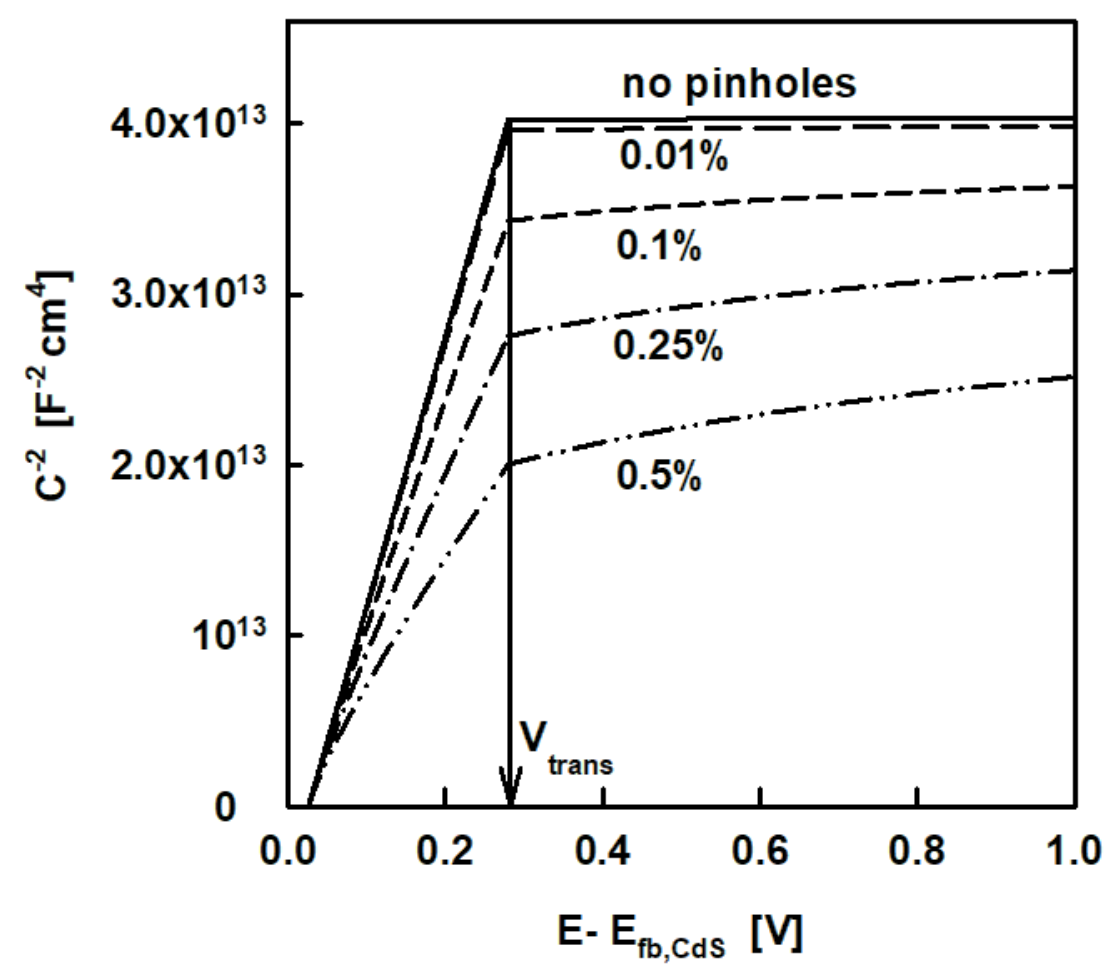

Figure 2. Calculated effect of pinholes on Mott Schottky plots for a $50 \mathrm{~nm}$ CdS film on FTO. $N_{d, C d S}=$ $10^{17} \mathrm{~cm}^{-3}, N_{d, F T O}=10^{21} \mathrm{~cm}^{-3}$. Pinhole coverage as shown. Note the decrease in initial slope and increase in slope beyond $V_{\text {trans }}$ as the pinhole coverage increases.

The plots show that the slope of the Mott Schottky plot below the transition voltage is decreased by pinholes, which will lead to overestimation of the doping density of the CdS (cf. equation 2). At the same time, the slope of the Mott Schottky plot above $V_{\text {trans }}$ become steeper as the pinhole coverage increases. Since in the case of a perfect pinhole-free film, the Mott Schottky plot becomes essentially horizontal past the transition voltage, any deviation from horizontal behaviour provides evidence for the presence of pin holes in the CdS film. Values of $\theta$ and the correct values of $N_{d, C d S}$ can be estimated by fitting the experimental Mott Schottky plots to the model. Values of the film thickness $d_{C d S}$ are obtained from the transition voltage, and initial values of the doping density $N_{d, C d S}$ from the slope of the Mott Schottky plot below $V_{\text {trans. }} N_{d, C d S}$ is then decreased as $\theta$ is increased from zero until an acceptable fit to both sections of the Mott Schottky plot is obtained. 


\section{Cyclic voltammetry with $\mathrm{Fe}(\mathrm{CN})_{6}^{4-}$}

Since oxidation of $\mathrm{Fe}(\mathrm{CN})_{6}{ }^{4-}$ ions cannot take place at n-type $\mathrm{CdS}$ in the dark, any oxidation current measured with an electrolyte containing $\mathrm{K}_{4} \mathrm{Fe}(\mathrm{CN})_{6}$ must arise from areas of FTO exposed by pinholes. These small areas will behave as ultramicroelectrodes [9], with diffusion of electroactive ions taking place in the hemispherical geometry illustrated in Figure 3.
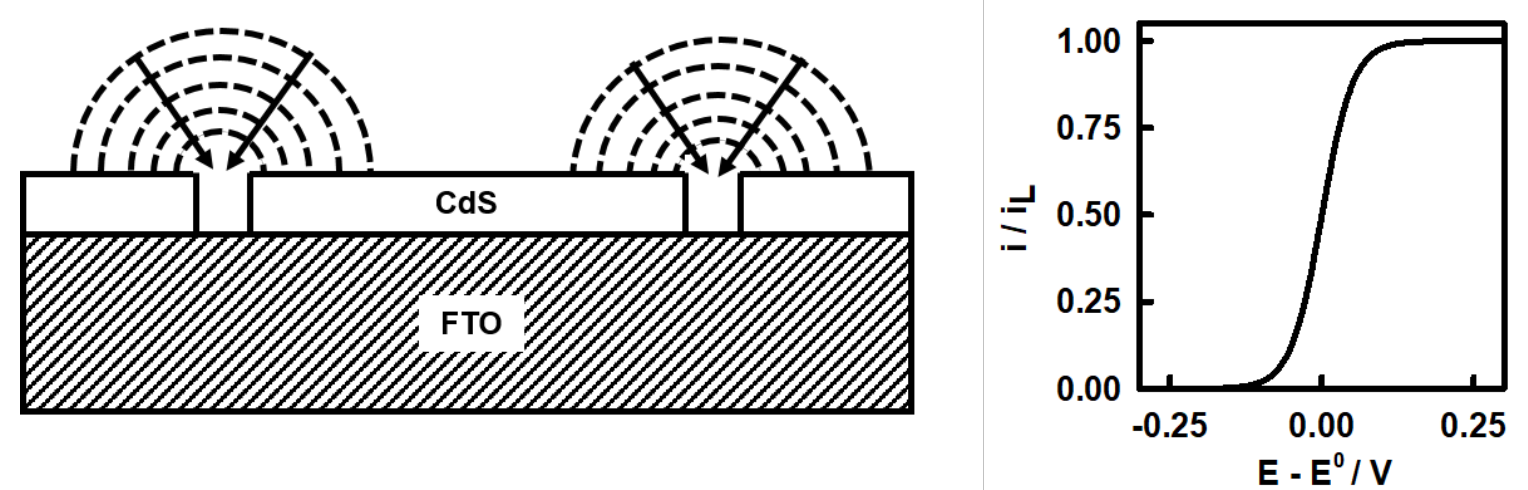

Figure 3. The formation of hemispherical diffusion zones enhances the flux of $\mathrm{Fe}(\mathrm{CN})_{6}{ }^{4-}$ to pinholes in CdS film on FTO, increasing the current density compared with planar diffusion. The current-voltage curve for the oxidation of $\mathrm{Fe}(\mathrm{CN})_{6}{ }^{4-}$ is $\mathrm{S}$-shaped, as shown on the right $\left(i_{L}\right.$ is the limiting plateau current: equation 8). Provided that the scan is slow, the current is the same on the forward and reverse scans. $E^{0}$ is the standard reduction potential of the hexacyanoferrate redox couple.

We now show that the fact that diffusion is hemispherical increases the sensitivity of voltammetry as a technique for detecting pinholes. The current voltage plot for a single circular ultramicroelectrode of radius $r$ is S-shaped, with a limiting steady state current $i_{L}$ given by

$$
i_{L}=4 r F D_{F e(C N)_{6}^{4-}} C_{F e(C N)_{6}^{4-}}
$$

Here $F$ is Faraday's constant and the $D$ and $C$ terms refer to the diffusion coefficient $\left(\mathrm{cm}^{2} \mathrm{~s}^{-1}\right)$ and concentration of the hexacyanoferrate ions $\left(\mathrm{mol} \mathrm{cm}^{-3}\right)$ respectively. Surprisingly, the current does not depend on the square of the radius as one might expect (the disc area is $\pi r^{2}$ ). Instead, it varies linearly with $r$. The current density $i_{L} / \pi r^{2}$ therefore increases as the pinholes become smaller. The limiting current for an array of $N_{p h}$ identical circular pinholes with radii $r$ is 


$$
i_{L}=4 N_{p h} r F D_{F e(C N)_{6}^{4-}} C_{F e(C N)_{6}^{4-}}
$$

We note in passing that the corresponding pinhole coverage, $\theta=N_{p h} \pi r^{2}$.

The limiting current given by equation 9 can be measured as a plateau using a slow voltage scan, as illustrated on the right-hand side of Figure 3. It is independent of sweep rate at low sweep rates because hemispherical diffusion sets up a steady state, which also means that the current is the same on the reverse sweep. This situation contrasts with the case of planar diffusion, where the diffusion layer thickness varies with time, and a reduction peak is seen on the reverse scan.

The peak current observed in cyclic voltammetry increases with the square root of the sweep rate $v$.

$$
i_{\text {peak }}=0.446 A F C_{F e(C N)_{6}^{4-}}\left(\frac{F D_{F e(C N)_{6}^{4-}}}{R T} v\right)^{1 / 2}
$$

In the experiments reported here, a sweep rate of $20 \mathrm{mV} \mathrm{s}^{-1}$ was used, and the concentration of $\mathrm{Fe}(\mathrm{CN})_{6}^{4-}$ was $5 \mathrm{mM}$. The currents predicted for the same area under conditions of hemispherical and planar diffusion are compared in Figure 4 for a total area of $10^{-3} \mathrm{~cm}^{2}$.

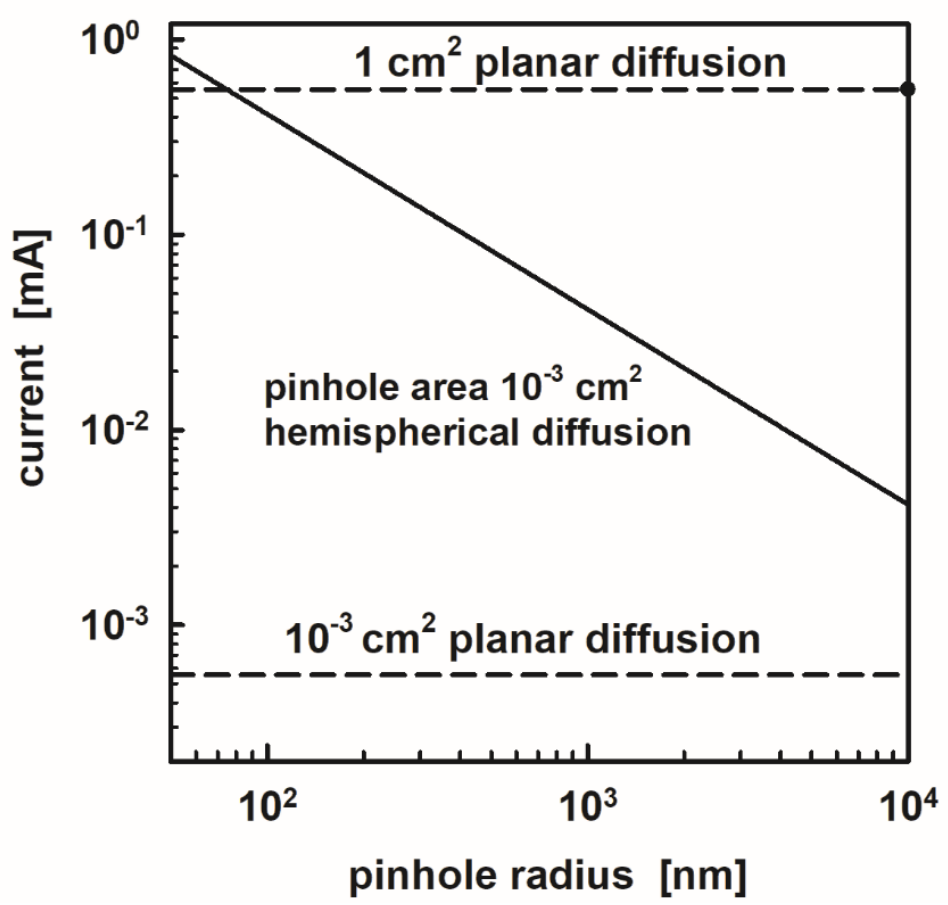

Figure 4. Plots comparing the $\mathrm{Fe}(\mathrm{CN})_{6}{ }^{4-}$ oxidation current predicted for the same area $\left(10^{-3} \mathrm{~cm}^{2}\right)$ in the case of planar diffusion (peak current for a sweep rate of $20 \mathrm{mV} \mathrm{s}^{-1}$ ) and hemispherical diffusion to an array of identical pinholes of radius $r$ (limiting plateau current). $\mathrm{Fe}(\mathrm{CN})_{6}{ }^{4-}$ concentration $5 \mathrm{mM}$. 
Diffusion coefficient of $\mathrm{Fe}(\mathrm{CN})_{6}{ }^{4-} 9 \times 10^{-6} \mathrm{~cm}^{2} \mathrm{~s}^{-1}$. The current predicted in the planar case for a $1 \mathrm{~cm}^{2}$ electrode is shown for comparison. The enhancement of the current for an array of pinholes increases as the pinhole size decreases, and for very small pores ( $r=100 \mathrm{~nm}$ or less), the current can even exceed the value for planar diffusion to an electrode with an area $10^{3}$ times larger (i.e. $1 \mathrm{~cm}^{2}$ in this case).

If the size distribution is narrow, the coverage of pinholes obtained by fitting the Mott Schottky plots can be used to estimate a mean pinhole size from the limiting voltammetric current. In this case, the mean pinhole radius $r_{\text {mean }}$ is given by

$$
r_{\text {mean }}=\frac{4 \theta F D_{F e(C N)_{6}^{4-}} C_{F e(C N)_{6}^{4-}}}{\pi i_{L}}
$$

\section{Experimental}

CdS films were deposited on $1 \mathrm{~mm}$ thick FTO-coatyed glass (TEC 15৫ Visiontek) at a temperature of $70^{\circ} \mathrm{C}$ using a water jacketed vessel, and an ultrasonic probe (Misonix ultrasonic mixing probe, power $10 \mathrm{~W}$ rms) was used to stir the solution. The FTO samples were ultrasonically cleaned in water/acetone and isopropyl alcohol followed by a 3-minute ozone treatment. The two different chemical bath compositions investigated have been used in thin film solar cell fabrication at CREST, Loughborough. However, the plasma cleaning step used routinely by us to reduce pinholes [1] was omitted. The compositions of the two baths are summarized below.

Bath A. $200 \mathrm{ml} \mathrm{H}_{2} \mathrm{O}, 35 \mathrm{ml} \mathrm{25 \%}$ ammonia, $20 \mathrm{ml} 0.1 \mathrm{M}$ thiourea, $20 \mathrm{ml} 0.01 \mathrm{M}$ cadmium acetate, $9 \mathrm{mg}$ cadmium chloride. $70^{\circ} \mathrm{C}$. Deposition time 30 minutes. Used for CdTe cells.

Bath B. $183 \mathrm{ml}$ water, $32.6 \mathrm{ml} 24 \%$ ammonia, $12.5 \mathrm{ml} 1.5 \mathrm{M}$ thiourea, $25 \mathrm{ml} \mathrm{0.015} \mathrm{M} \mathrm{cadmium}$ sulfate. $70^{\circ} \mathrm{C}$. Deposition time 15 minutes. Used for CIGS cells.

The amount of CdS deposited on the FTO substrates was determined by dissolving a defined area of the films in nitric acid and measuring the cadmium content by atomic absorption spectroscopy (AAS). The film thickness values obtained were $49 \mathrm{~nm}$ for bath A and $75 \mathrm{~nm}$ for 
bath B. No correction was made for surface roughness. SEM images were acquired with an JEOL JSM-7800F Field Emission Scanning Electron Microscope (FESEM) operating at 5kV.

For impedance and voltammetric measurements with electrolyte contacts, the CdScoated samples were contacted by etching away as small area of CdS at the top of the sample with $\mathrm{HCl}$ to expose the $\mathrm{FTO}$, and $\mathrm{Kapton}(\mathrm{C}$ tape was used to mask off a defined area of $\mathrm{CdS}$ film of around $3.5 \mathrm{~cm}^{2}$. Impedance and voltammetry measurements on bare FTO were made with smaller areas. To prevent any dissolution of the CdS films, a neutral buffer solution was used for the impedance measurements (50 mM phosphate buffer, $\mathrm{pH}$ 7). A $1 \mathrm{M} \mathrm{KNO}_{3}$ solution with $5 \mathrm{mM} \mathrm{K}_{4} \mathrm{Fe}(\mathrm{CN})_{6}$ was used for the voltammetric measurements. The three-electrode glass cell comprised a platinum counter electrode, a silver chloride reference electrode $(\mathrm{Ag} / \mathrm{AgCl})$ and the CdS or FTO working electrode.

Frequency-resolved impedance measurements were made over the range $10 \mathrm{~Hz}$ to 10 $\mathrm{kHz}$ using an Autolab PGSTAT12 potentiostat, typically at intervals of 20 or $50 \mathrm{mV}$ over the range of interest. In order to extract the capacitance, ZView software (Scribner) was used to fit impedance spectra to an equivalent circuit consisting of a series resistance connected to parallel RC circuit.

\section{Results and discussion}

Figure 5 compares FESEM micrographs of bare FTO with CdS-coated FTO (in this case the CdS was deposited with bath B). The underlying morphology of the FTO is still visible after CdS deposition. The CdS layer is composed of very small crystallites that appear to conformally coat the larger features of the FTO substrate. Cross-sectional images show the CdS layer with reasonable contrast, but accurate measurement of the layer thickness was made difficult by the morphology of the FTO. FESEM images for films deposited using bath A showed no obvious differences. 

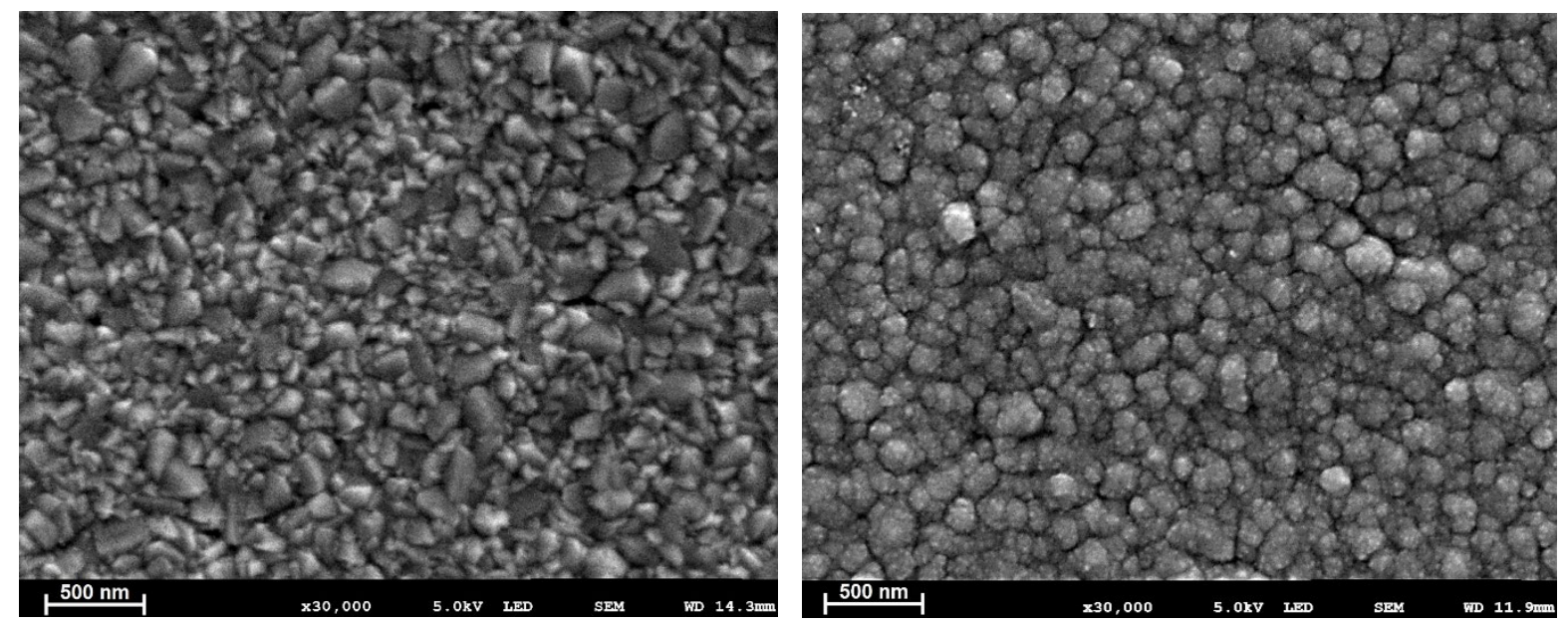

Figure 5. FESEM images of bare FTO substrate 15 (left) and of CdS-coated FTO 15 (right). The CdS film in this case was grown using Bath B. The scale bar shown is $100 \mathrm{~nm}$.

Figure 6 shows the experimental Mott Schottky plot and the fit for a CdS layer grown using Bath A. Note that the plot does not show a transition to a horizontal region above the transition voltage. Instead the plot continues to slope upwards beyond the transition voltage, indicating significant porosity. This conclusion is confirmed by the fitting, which gives a fractional pinhole coverage of $0.42 \%$ 


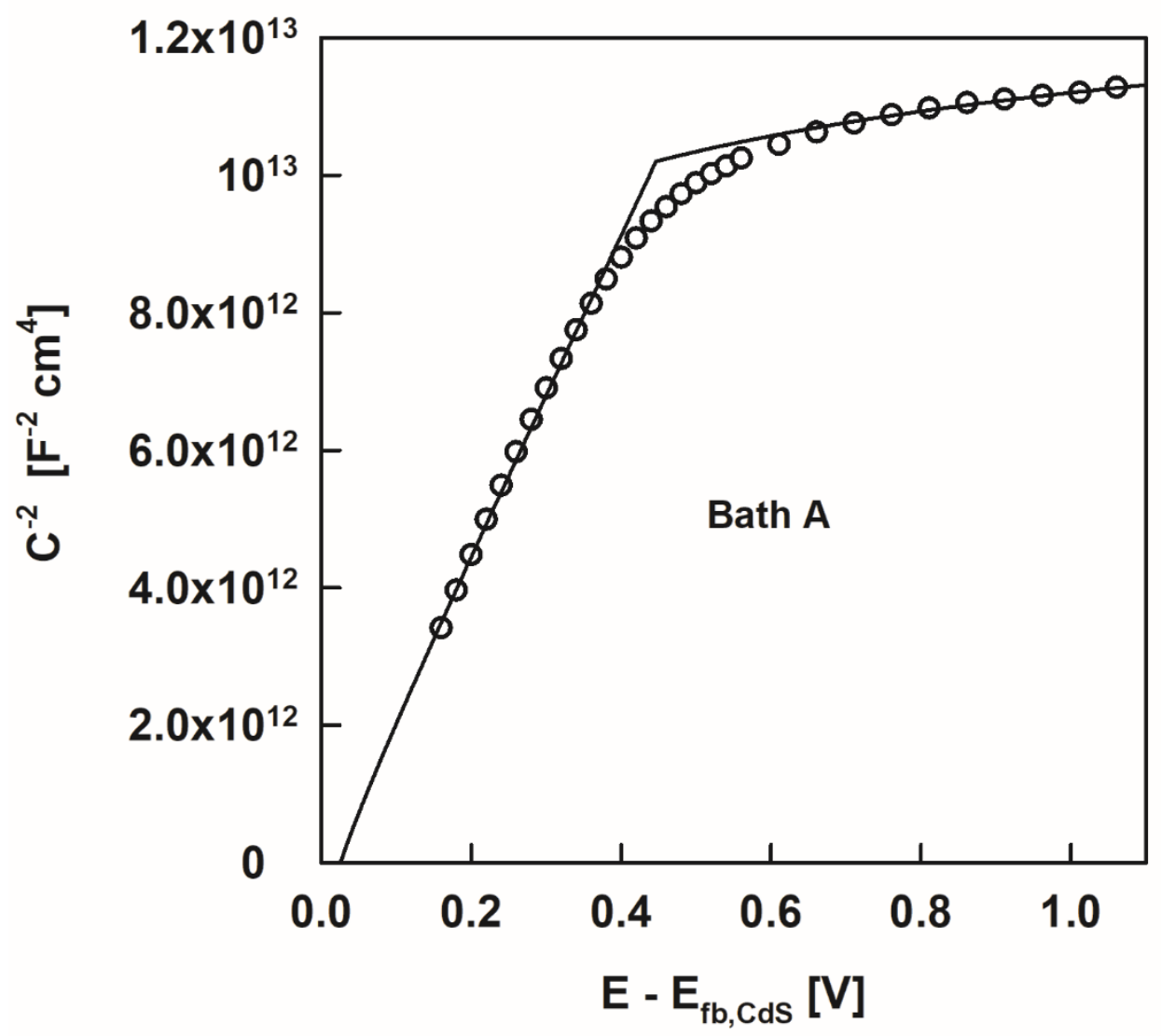

Figure 6. Circles - experimental Mott Schottky plot for a CdS film grown in Bath A. The voltage axis $E-E_{f b}$ was calculated using the measured flatband potential of $-0.548 \mathrm{~V}$ vs. $\mathrm{Ag} / \mathrm{AgCl}$. Lines - fit to pinhole model. The fit gives $d_{C d S}=30 \mathrm{~nm}, N_{d, C d S}=4.6 \times 10^{17} \mathrm{~cm}^{-3}, \theta=0.42 \%$.

If the effect of pinholes is neglected, the CdS doping density obtained from the initial slope of the Mott Schottky plot is $6.9 \times 10^{17} \mathrm{~cm}^{-3}$. However, fitting taking into account the effect of pinholes gives a lower value of $N_{d, C d S}=4.6 \times 10^{17} \mathrm{~cm}^{-3}$. It should be noted that the surface roughness of the FTO substrate has not been considered in this calculation. Since the thin CdS film is conformal on the larger length scale of the FTO crystallites, the measured capacitance values should be divided by a surface roughness factor $\mathfrak{R}>1$. The doping densities derived from the Mott Schottky plots should therefore be divided by $\mathfrak{R}^{2}$, which means that they will be a factor of 2 to 3 times lower than the reported values (corresponding to $1.4<\Re<1.7$ ). 
The value of the CdS film thickness obtained from the fitting $(30 \mathrm{~nm})$ is lower than the value derived from the AAS measurements $(49 \mathrm{~nm})$. There are two possible reasons for the difference. The first is that the surface roughness of the FTO substrate has not been accounted for in the capacitance measurements, nor in the calculation of film thickness from the AAS data. The second is that the analysis assumes that the CdS film is compact throughout and behaves as a bulk semiconductor. In fact. the work of the Lincot group [5] suggests that CdS films can have a bilayer structure, with a compact inner layer and a more porous outer layer. In this case, only the inner layer would behave as a dielectric.

Figure 7 shows the Mott Schottky plot for a CdS film grown in bath B. In this case, a transition to a horizontal plateau is observed above the transition voltage, indicating lower pinhole coverage. This conclusion is confirmed by the fitting, which gives a pinole coverage below $0.05 \%$. This is close to the lower limit for the fitting, i.e. the films are almost pinhole free. The fitting also shows that the CdS film grown in bath $\mathrm{B}$ has an order of magnitude lower doping density than the film grown in bath $\mathrm{A}$, where $\mathrm{CdCl}_{2}$ was added to improve the conductivity. The film thickness derived from the fit $(64 \mathrm{~nm})$ is again lower than the value derived from the AAS measurements $(75 \mathrm{~nm})$, but this is not unexpected for the reasons outlined above. 


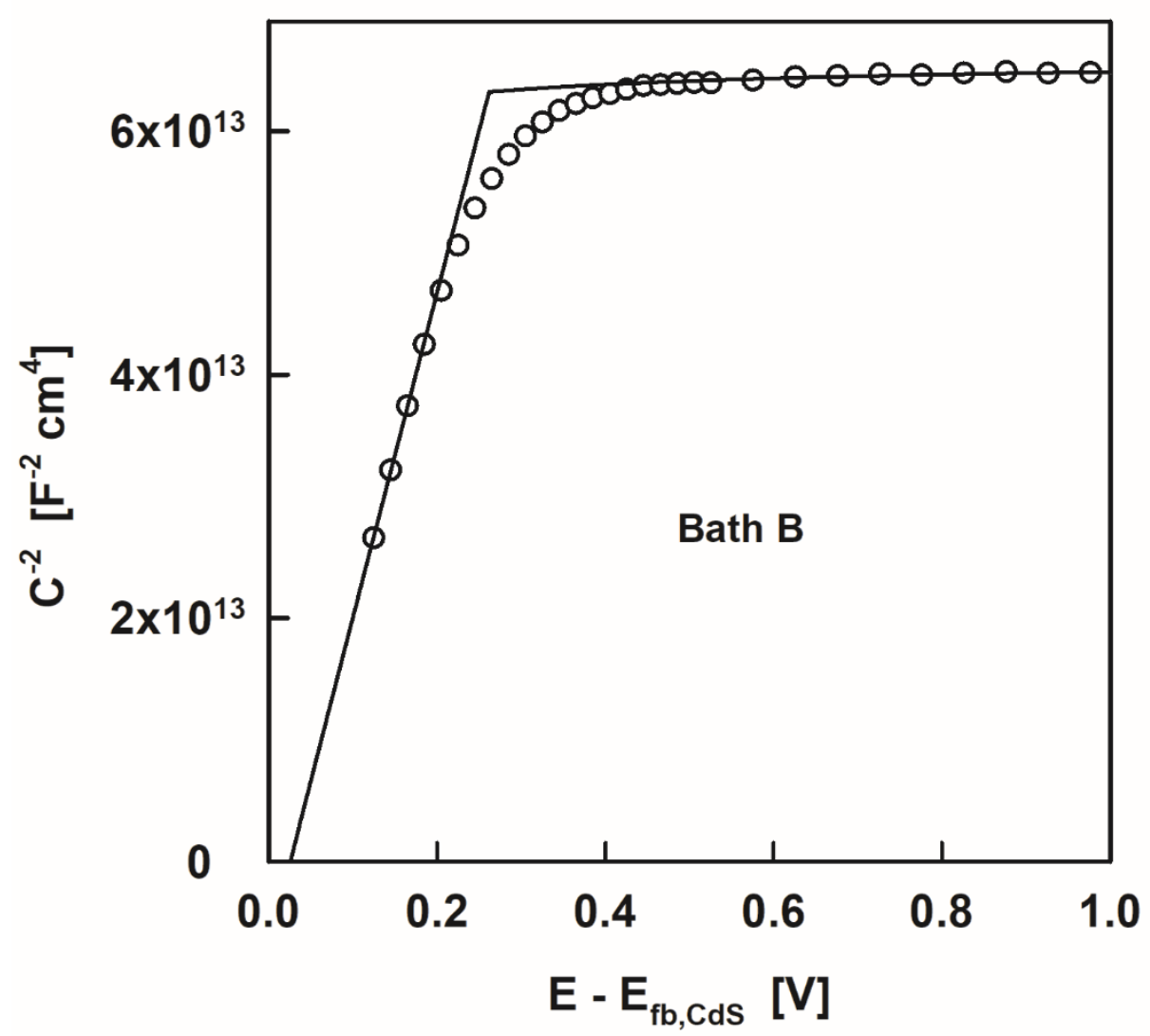

Figure 7. Circles - experimental Mott Schottky plot for a CdS film grown in Bath B. The voltage axis $E-E_{f b}$ was calculated using the measured flatband potential of $-0.505 \mathrm{~V}$ vs. $\mathrm{Ag} / \mathrm{AgCl}$. Note the transition to a horizontal plateau, which indicates low pinhole coverage Lines - fit to pin hole model. The fit gives $d_{C d S}=64 \mathrm{~nm}, N_{d, C d S}=5.6 \times 10^{16} \mathrm{~cm}^{-3}, \theta<0.05 \%$.

These results give a clear indication that the $\mathrm{CdS}$ films grown in bath $\mathrm{A}$ have more pinholes or porosity than those grown in bath B. Cyclic voltammetry confirms this conclusion. Figure 8 compares the current voltage responses measured for the two CdS films in the solution containing $\mathrm{Fe}(\mathrm{CN})_{6}{ }^{4-}$ ions. The cyclic voltammogram obtained using bare FTO has been included to aid comparison (note the different scales for the current axes). The values of $i_{L}$ at the positive limit of the scan $(1.0 \mathrm{~V}$ vs. $\mathrm{Ag} / \mathrm{AgCl})$ are $15.9 \mu \mathrm{A} \mathrm{cm}{ }^{-2}$ and $1.06 \mu \mathrm{A} \mathrm{cm}^{-2}$ respectively for the films grown in baths $\mathrm{A}$ and $\mathrm{B}$. 


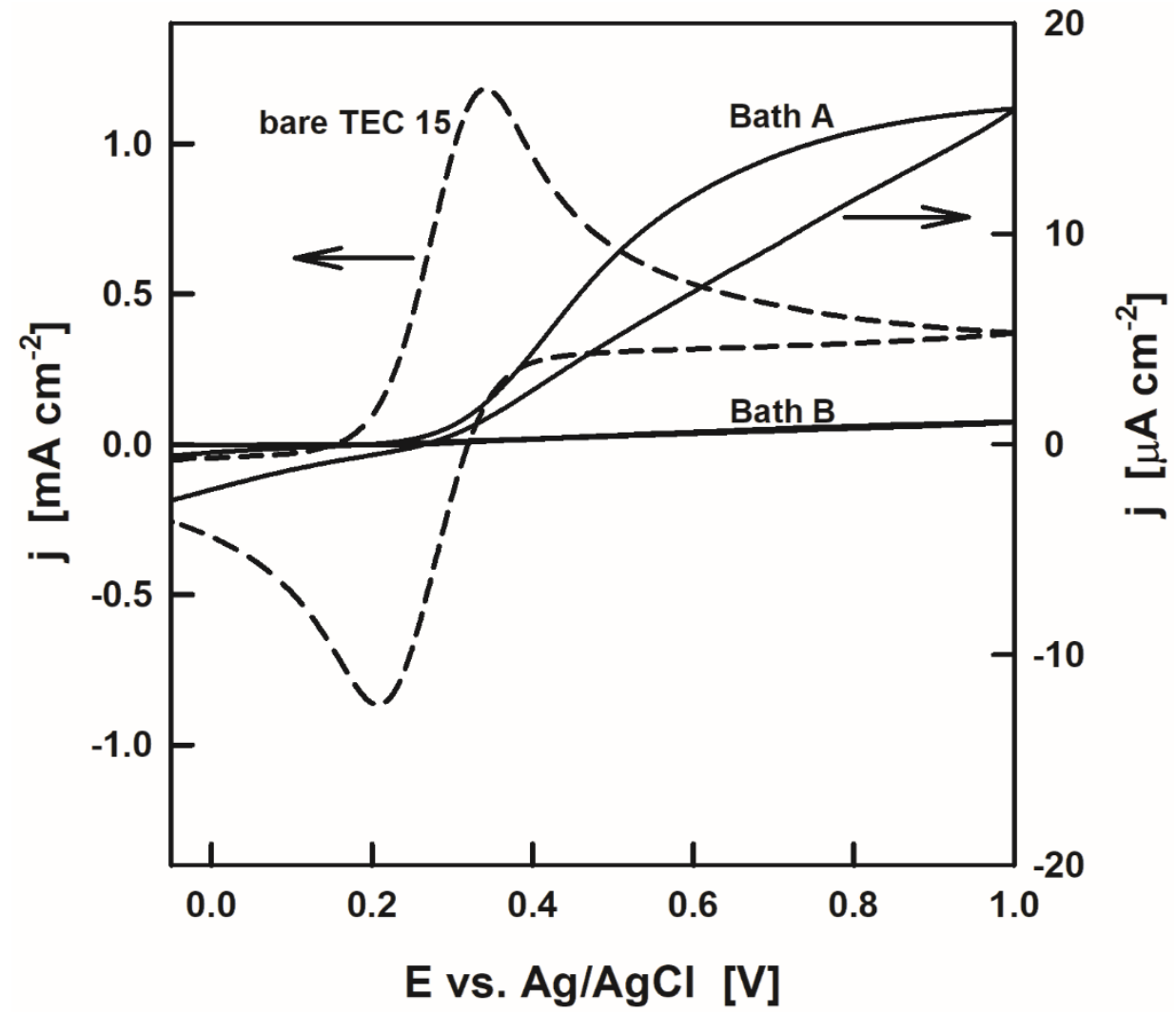

Figure 8. Comparison of voltammograms obtained with bare FTO (left hand current scale) and CdS films grown in baths $\mathrm{A}$ and $\mathrm{B}$ (right hand current scale). Solution $1 \mathrm{M} \mathrm{KNO}_{3}+5 \mathrm{mM} \mathrm{K}_{4} \mathrm{FE}(\mathrm{CN})_{6}$. Sweep rate $20 \mathrm{mV} \mathrm{s}^{-1}$. Note the very low oxidation current in the case of the film grown in Bath B $\left(i_{L}=\right.$ ca.1 $\mu \mathrm{A})$ compared with Bath AS $\left(i_{L}\right.$ ca $\left.16 \mu \mathrm{A}\right)$. This is consistent with the very low pinhole coverage estimated for Bath B from the capacitance measurements.

It is tempting to estimate the pinhole coverage from the ratio of the currents measured with and without the CdS film. However, as pointed out above, this ratio depends on the sweep rate since the peak current varies with the square root of the sweep rate in the case of planar diffusion, whereas the steady state plateau current for an array of ultramicroelectrodes should be independent of sweep rate. This current depends not only on pinhole coverage but also on the pinhole sizes as a consequence of the hemispherical diffusion geometry. The ratio of the limiting currents measured for the two films $\left(j_{A} / j_{B}=15\right)$ is reasonably consistent with the ratio of pinhole coverages estimated from the capacitance measurements $\left(\theta_{\mathrm{A}} / \theta_{\mathrm{B}}>8\right)$, suggesting that the pinhole sizes in the two cases are not too dissimilar. 
The shape of the voltammetric response for the two films is more drawn out than expected for facile oxidation of $\mathrm{Fe}(\mathrm{CN})_{6}{ }^{4-}$. The S-shaped $i V$ curve in Figure 3 is calculated assuming that electron transfer is rapid. This is usually the case for the oxidation of $\mathrm{Fe}(\mathrm{CN})_{6}{ }^{4-}$ at metal electrodes or FTO. The more drawn out shape observed for the CdS-coated films suggests sluggish electron transfer. This could indicate that the bottom of the pinholes does not expose pristine FTO. Instead a very thin layer - perhaps even a monolayer - of CdS may remain, so the electrons must tunnel through a barrier for oxidation to occur.

As pointed out above, estimation of the mean pore size from $\theta$ and the measured currents using equation 11 is only possible if the pore size distribution is narrow. Nevertheless, the calculation could give an idea of whether we are dealing with a small number of large pores or many small pores. $r_{\text {mean }}$ calculated for the film grown in bath $\mathrm{A}\left(i_{L}=15.9 \mu \mathrm{A}\right)$ is 15 microns, and for the film grown in bath $\mathrm{B}\left(i_{L}=1.06 \mu \mathrm{A}\right)$ it is 26 microns. This suggests that the main difference between the films is in the number of pores rather than their sizes. If we assume a narrow pore distribution, we obtain around 600 pores $\mathrm{cm}^{-2}$ for the film grown in bath $\mathrm{A}$, but fewer than $25 \mathrm{~cm}^{-2}$ for bath $\mathrm{B}$. Of course, the assumption of a narrow distribution is unjustified, but nevertheless the result is interesting. Work is in progress to try to relate these results to pore sizes and number densities obtained by optical and scanning electron microscopy.

\section{Conclusions}

This study using electrolyte contacts has revealed diagnostic criteria for identifying pinholed films from Mott Schottky plots for CdS films on FTO. The analysis of the capacitance voltage characteristics shows that it is possible to estimate the total area of FTO exposed by pinholes by fitting the Mott Schottky plots to a simple model. The study also explores the use of electroactive species $\left(\mathrm{Fe}(\mathrm{CN})_{6}{ }^{4-}\right)$ to detect pinholes and highlights the need to consider the diffusion geometry when analysing the measured current response. The methods should prove 
useful for rapid non-destructive assessment of the quality of CdS buffer layers prepared by different routes. Finally, we note that absorber layers such as CIGS can be characterized using strongly the strongly oxidizing hexacyanoferrate redox system provided that a protective $\mathrm{CdS}$ layers is present to prevent hole injection [10].

\section{Acknowledgments}

This work was supported by the UK Engineering and Science Research Council (EPSRC): PVTeam Grant No. EP/L017792/1.

\section{References}

[1] F. Lisco, A. Abbas, B. Maniscalco, P.M. Kaminski, M. Losurdo, K. Bass, G. Claudio, J.M. Walls, Pinhole free thin film CdS deposited by chemical bath using a substrate reactive plasma treatment, Journal of Renewable and Sustainable Energy, 6 (2014) 011202.

[2] M. Tashkandi, Pinholes and morphology of CdS films: The effect on the open circuit voltage of CdTe solar cells, PhD thesis, Dept. Mech. Eng., Colorado State Univ, Fort Collins, CO, USA, 2012, 2012.

[3] R. Ortega-Borges, D. Lincot, Mechanism of Chemical Bath Deposition of Cadmium Sulfide Thin Films in the Ammonia-Thiourea System: In Situ Kinetic Study and Modelization, Journal of The Electrochemical Society, 140 (1993) 3464-3473.

[4] M. Froment, D. Lincot, Phase formation processes in solution at the atomic level: Metal chalcogenide semiconductors, Electrochimica Acta, 40 (1995) 1293-1303.

[5] D. Lincot, R.O. Borges, Chemical Bath Deposition of Cadmium Sulfide Thin Films. In Situ Growth and Structural Studies by Combined Quartz Crystal Microbalance and Electrochemical Impedance Techniques, Journal of The Electrochemical Society, 139 (1992) 1880-1889. 
[6] M.E. Ozsan, D.R. Johnson, M. Sadeghi, D. Sivapathasundaram, G. Goodlet, M.J. Furlong, L.M. Peter, A.A. Shingleton, Optical and electrical characterization of CdS thin films, Journal of Materials Science-Materials in Electronics, 7 (1996) 119-125.

[7] R. van de Krol, A. Goossens, J. Schoonman, Mott-Schottky Analysis of Nanometer-Scale Thin-Film Anatase TiO2, Journal of The Electrochemical Society, 144 (1997) 1723-1727.

[8] A.P. Samantilleke, M.F. Cerqueira, S. Heavens, P. Warren, I.M. Dharmadasa, G.E.A. Muftah, C.J.R. Silva, B. Marí, Characterisation of chemical bath deposited CdS thin films on different substrates using electrolyte contacts, Thin Solid Films, 519 (2011) 75837586.

[9] H. Jürgen, Ultramicroelectrodes in Electrochemistry, Angewandte Chemie International Edition in English, 32 (1993) 1268-1288.

[10] D. Colombara, P.J. Dale, G.P. Kissling, L.M. Peter, S. Tombolato, Photoelectrochemical Screening of Solar Cell Absorber Layers: Electron Transfer Kinetics and Surface Stabilization, The Journal of Physical Chemistry C, 120 (2016) 15956-15965. 